\title{
A Single Dose, Randomized, Controlled Proof-Of-Mechanism Study of a Novel Vasopressin la Receptor Antagonist (RG77I3) in High-Functioning Adults with Autism Spectrum Disorder
}

\author{
Daniel Umbricht*, , Marta del Valle Rubido', Eric Hollander², James T McCracken ${ }^{3}$, Frederick Shic ${ }^{4}$, \\ Lawrence Scahill ${ }^{5}$, Jana Noeldeke', Lauren Boak ${ }^{6}$, Omar Khwaja', Lisa Squassante', \\ Christophe Grundschober', Heidemarie Kletzl' and Paulo Fontoura ${ }^{6}$ \\ 'Roche Pharmaceutical Research and Early Development, Roche Innovation Center, F. Hoffmann - La Roche, Ltd., Basel, Switzerland; '2Department \\ of Psychiatry and Behavioral Sciences, Albert Einstein College of Medicine, Montefiore Medical Center Bronx, New York, NY, USA; ${ }^{3}$ Psychiatry and \\ Biobehavioral Sciences, CART, UCLA Semel Institute, Los Angeles, CA, USA; ${ }^{4}$ Child Study Center, Yale University, New Haven, CT, USA; 5 Department \\ of Pediatrics, Emory University, Marcus Autism Center, Atlanta, GA, USA; ${ }^{6}$ Roche Product Development Neuroscience, Basel, Switzerland
}

\begin{abstract}
The core symptoms of autism spectrum disorder (ASD) include impaired social communication, repetitive behaviors, and restricted interests. No effective pharmacotherapy for these core deficits exists. Within the domain of social communication, the vasopressin system is implicated in social cognition and social signaling deficits of ASD, and represents a potential therapeutic target. We assessed the effects of a single $20 \mathrm{mg}$ intravenous dose of the arginine vasopressin receptor IA (VIa) antagonist, RG77I3, on exploratory biomarkers (eye tracking), behavioral and clinical measures of social cognition and communication (affective speech recognition (ASR), reading the mind in the eyes, olfactory identification, scripted interaction), and safety and tolerability in a multicenter, randomized, double-blind, placebocontrolled, cross-over study of 19 high-functioning adult male subjects with DSM-IV Autistic Disorder (age I8-45 years; full scale IQ > 70; $A B C$-Irritability subscale $\leqslant 13)$. Eye-tracking showed an increase in biological motion orienting preference with $R G 77 \mid 3$ (ES $=0.8$, $p=0.047)$ and a non-significant improvement in the composite score $(E S=0.2, p=0.29) . R G 77 \mid 3$ reduced ability to detect lust $(E S=-0.8, p=0.03)$ and fear $(E S=-0.7, p=0.07)$ in ASR. However, when all eight individual emotion subscales were combined into an overall ASR performance score, the reduction was non-significant $(E S=-0.1, p=0.59)$. Thirteen adverse events were reported in 10 subjects; all were of mild ( I // 3) or moderate (2/13) severity. Although interpretation should be cautious due to multiple comparisons and small sample size, these results provide preliminary evidence from experimental and behavioral biomarkers, that blockade of the $\mathrm{VIa}$ receptor may improve social communication in adults with high-functioning ASD. ClinicalTrials.gov identifier: NCTOI 474278 A Study of RO5028442 in Adult Male High-Functioning Autistic Patients. Available at: https://clinicaltrials.gov/ct2/show/NCT0 474278

Neuropsychopharmacology (2017) 42, 1914-1923; doi:10.1038/npp.2016.232; published online 16 November 2016
\end{abstract}

\section{INTRODUCTION}

Autism spectrum disorder (ASD) is a heterogeneous developmental condition characterized by impairment in reciprocal social behavior and communication (social communication), and repetitive behaviors and restricted interests (restricted, repetitive behaviors) (American Psychiatric Association, 2013). In the American Psychiatric Association's most recent diagnostic guidelines (DSM-V), the term ASD unifies former separate diagnostic categories of Autistic

* Correspondence: Dr D Umbricht, Roche Innovation Center, Basel, F. Hoffmann-La Roche Ltd, Grenzacherstrasse 124, Basel 4070, Switzerland, Tel: +4I 6 I 688 3043, Fax: +4I 6 I 6879848 ,

E-mail: daniel.umbricht@roche.com

Received 9 June 2016; revised 12 September 2016; accepted 17 September 2016; accepted article preview online 6 October 2016
Disorder with Asperger's Disorder, and Pervasive Developmental Disorder-Not Otherwise Specified (PDD-NOS), capturing the spectrum of features and impairment (American Psychiatric Association, 2013). The estimated prevalence of ASD is 7.6 per 1000 (1/132) persons worldwide, equivalent to 52 million cases in 2010 (Baxter et al, 2015; Centers for Disease Control, 2014). Current pharmacological treatments for ASD commonly include antipsychotics, antidepressants, and psychostimulants, targeting associated symptoms such as aggression, self-injurious behavior, impulsivity and irritability, stereotypies, hyperactivity, anxiety and mood symptoms, but no efficacious pharmacotherapy for the core symptoms of ASD exists (Ji and Findling, 2015). Consequently, there is a critical need for treatments that target the lifelong burden of core deficits of social communication and restricted repetitive behaviors. 
Social cognition is an integral part of social communication, comprising perception and processing of socially salient, visual and auditory information, and also higher order integrative functions including empathy and theory of mind (Baron-Cohen et al, 1985; Frith and Frith, 2005). ASDrelated differences have been identified at multiple levels of social cognition and communication. The oxytocin and vasopressin systems play a critical role in social cognition and social signaling deficits of ASD, and may be promising therapeutic targets (Andari et al, 2010; Hollander et al, 2007). The importance of the vasopressin system in social cognition and behavior is highlighted by studies of variants of the arginine vasopressin receptor 1A (AVPR1A) gene, which encodes the V1a receptor. These studies have shown AVPR1A gene variants to be associated with autism (Kim et al, 2002; Wassink et al, 2004; Yirmiya et al, 2006; Yang et $a l, 2010$ ) and to modulate amygdala activation during emotional face processing (Meyer-Lindenberg et al, 2009). AVPR1A gene variants have also been associated with specific personality traits such as pair-bonding behavior in healthy volunteers (Meyer-Lindenberg et al, 2009; Walum et al, 2008). Furthermore, intranasal administration of vasopressin modulates a network involved in processing emotional information (the medial prefrontal cortex-amygdala circuitry) (Zink et al, 2010), and has been shown to increase threat perception in healthy volunteers (Thompson et al, 2004), and to impair emotion recognition in males (Uzefovsky et al, 2012). Taken together, modulation of vasopressin signaling represents a potential therapeutic target in ASD.

RG7713 is a potent and highly selective V1a receptor antagonist, which may provide a novel approach to treat core deficits of ASD (Ratni et al, 2015). A phase I single-ascending dose (SAD) study of RG7713 (intravenous administration) was previously conducted in healthy volunteers in order to provide safety, tolerability, and pharmacokinetic information. The SAD study confirmed brain penetration of RG7713 by measuring its concentration in the cerebrospinal fluid (CSF), and indicated that the highest dose tested, $20 \mathrm{mg}$, provides V1a receptor occupancy in the CNS of approximately $90 \%$ for at least $8 \mathrm{~h}$, with no safety concerns (a summary of pharmacokinetic data is presented in Supplementary Table S1) (F. Hoffmann-La Roche, 2015). Dose selection for clinical studies was based on the results of dose-response studies in the rat valproate model of autism, which demonstrated a maximum efficacy at $86 \%$ receptor occupancy. In addition, PET studies of different marketed GPCR antagonists have demonstrated that a receptor occupancy of $60-80 \%$ is needed for clinical efficacy (Grimwood and Hartig, 2009). Thus, we chose a single intravenous $20 \mathrm{mg}$ dose of RG7713 to explore its effects on exploratory behavioral biomarkers of social cognition and behavioral and clinical measures of social cognition and communication in high functioning adult subjects with autistic disorder. Safety and tolerability of RG7713 were also assessed.

\section{MATERIALS AND METHODS}

This study (NCT01474278) was a multicenter (three sites), randomized, double-blind, placebo-controlled, two period crossover study of a single dose of RG7713 in adult male subjects with high-functioning ASD $(n=19)$. The study was conducted under an experimental IND (which limited the number of participants and the duration of treatment) and in accordance with the Declaration of Helsinki and Good Clinical Practice guidelines (International Conference on Harmonisation, 1996; World Medical Association, 2008). The study protocol and all accompanying documentation were approved by institutional review boards (IRBs) at UCLA (UCLA IRB, Los Angeles, CA, USA), Yale University (Yale Human Investigation Committee, New Haven, CT, USA), and Albert Einstein College of Medicine (Albert Einstein West Campus IRB, Bronx, NY, USA). All participants provided written informed consent for involvement in the study.

A screening examination was performed within 5 weeks before the start of the study and included electrocardiograms (ECG), vital signs, physical examination, laboratory testing (hematology, chemistry, urinalysis, serology, and alcohol and drugs of abuse), clinical evaluation by clinicians with expertise in ASD diagnosis and treatment, and assessments with the Autism Diagnostic Observation Schedule (ADOS) (Lord et al, 1989), Wechsler Abbreviated Scale of Intelligence-Second Edition (WASI-2) (Wechsler, 1999), Columbia-Suicide Severity Rating Scale (C-SSRS) (Posner et al, 2011), Aberrant Behavior Checklist (ABC) (Aman et al, 1985), and Vineland Adaptive Behavior Scales-Second Edition (VABS-II) (Sparrow et al, 2005). Key inclusion and exclusion criteria are presented in Supplementary Table S2.

A single $20 \mathrm{mg}$ dose of RG7713 or placebo was administered intravenously as a $2-\mathrm{h}$ infusion to each subject on two different days (treatment visits 1 and 2) separated by a 7-14day washout period (Figure 1). Based on the pharmacokinetics observed in the SAD study (half-life $=7.5-9 \mathrm{~h}$ ), this washout period between administration of RG7713 and placebo was considered sufficient to avoid carryover effects. Subjects received RG7713 and placebo in one of two sequences: Sequence 1-subjects received $20 \mathrm{mg}$ RG7713 during treatment visit 1 followed by placebo during treatment visit 2 and Sequence 2-subjects received placebo followed by RG7713. Subjects were randomized to sequence 1 or 2 using a randomized treatment schedule that was developed by the study sponsor for each site and incorporated into double-blind (investigator and subject) treatment labeling.

During each treatment visit, the investigator carried out baseline assessments and checks before administration of study drug. Efficacy assessments-key exploratory behavioral biomarkers, behavioral and clinical measures of social cognition and communication, an abbreviated version of ABC, Clinical Global Impression-Improvement scale (CGI-I) (Guy, 1976) and State-Trait Anxiety Inventory (STAI) (Spielberger and Gorsuch, 1983)-were performed before and after, or after drug administration, as shown in Figure 1. Safety assessments included physical examination, review of adverse events (AEs), C-SSRS, ECG, vital signs and laboratory tests, also obtained pre- and post-infusion.

Efficacy assessments were selected on the basis of two key considerations. Firstly, to provide an evaluation of potential drug effects at different system levels of social cognition and communication, including a basic level of screening and acquiring socially relevant information (eye tracking), 


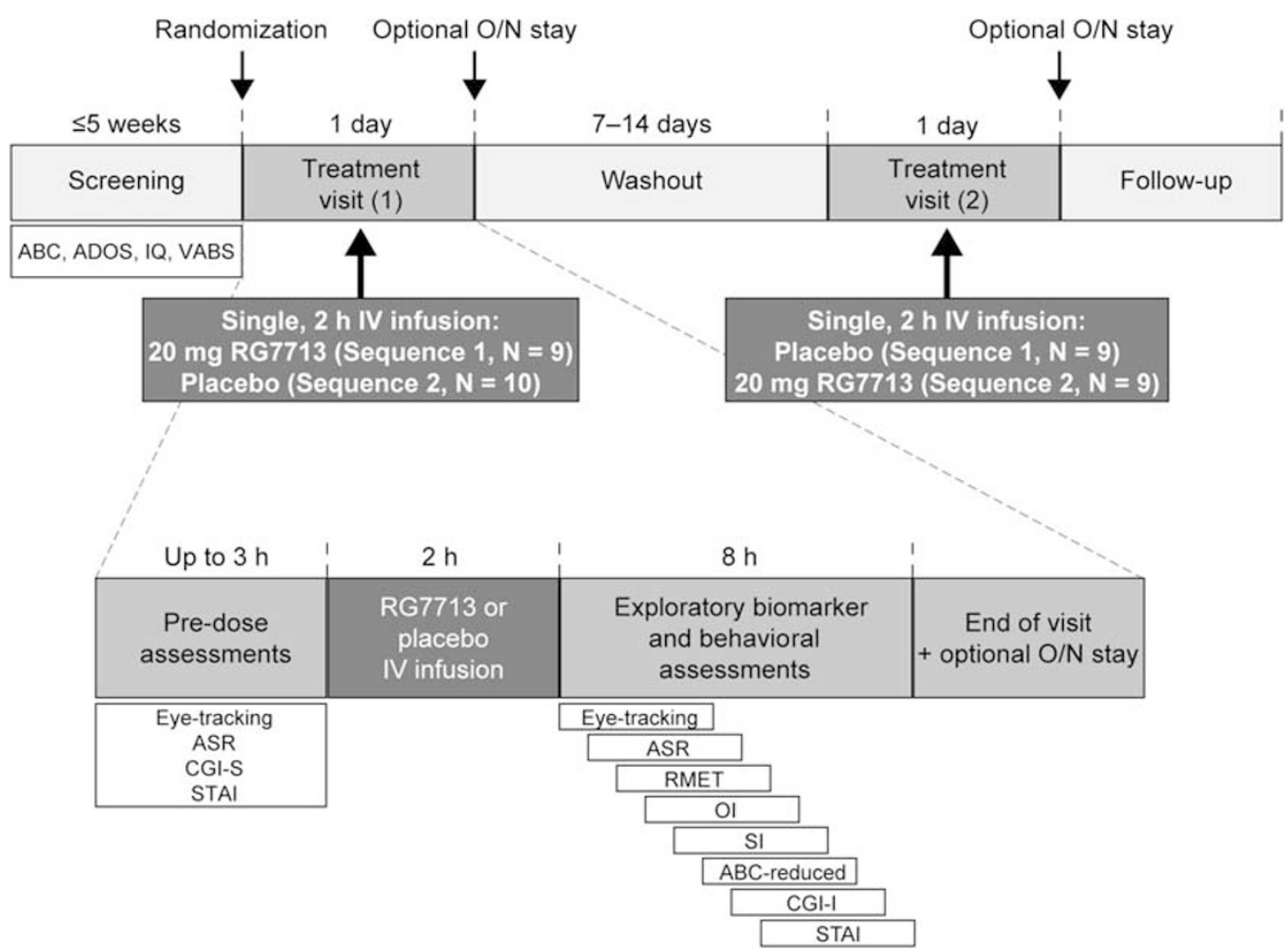

Figure I Study design. Abbreviations: ABC, Aberrant Behavior Checklist; ADOS, Autism Diagnostic Observation Schedule; ASR, affective speech recognition; CGI-I, Clinical Global Impressions - improvement; CGI-S, Clinical Global Impressions_-severity; h, hour; IV, intravenous; IQ, intelligence quotient; $\mathrm{OI}$, olfactory identification; O/N, overnight; RMET, reading the mind in the eyes test; SI, scripted interaction; STAI, State-Trait Anxiety Inventory; VABS, Vineland Adaptive Behavior Scale.

and intermediate levels corresponding to the ability to capture composite information that is critical for social communication (ASR, RMET) which would be mirrored in behavioral aspects of social communication (ABC, scripted interaction). Secondly, the choice of assessments was based on previous evidence of sensitivity to pharmacological intervention. This second consideration was only fulfilled for eye tracking, with these measures previously shown to be sensitive to administration of oxytocin (Andari et al, 2010).

An abbreviated version of the $\mathrm{ABC}$ was used as some of the items of this scale pertain to behaviors that can only be observed in a social context and were deemed inappropriate to assess the short-term effects of a pharmacological intervention. The items were selected by the investigators in this study (EH, JTM, LS) and included items 2, 4, 9, 10-12, $14,16,17,19,20,22,23,27,29,33,35-37,40-43,45-47,49$, and 58 from the 58-item full scale (Aman et al, 1985).

\section{Eye-Tracking}

A Tobii T60XL $60 \mathrm{~Hz}$ eye-tracking system was used to measure gaze position and pupil size in the following paradigms shown to differ between subjects with ASD and healthy volunteers: patterns of scanning during free-viewing of two actresses engaged in simple play activities (activity monitoring) (Shic et al, 2011); detection of biological motion $v s$ phase-scrambled biological motion (biodetection) (Kaiser et al, 2010); preference for biological motion over phase-scrambled biological motion or repetitive motion (biomotion) (Klin et al, 2009); gender discrimination; gaze direction discrimination (Andari et al, 2010); preference for dynamic human activities over dynamic geometric patterns (human activity) (Pierce et al, 2011); patterns of scanning during free-viewing of complex social scenes (clips from the movie Who's Afraid of Virginia Woolf?; WAVW) (Klin et al, 2002). An a priori defined composite eye-tracking score was derived from key parameters of all administered eye-tracking tasks, as a weighted sum of subcomposite scores (each subcomposite score indexing a different eye-tracking task). Further details of the components and calculations used for the composite score are provided in Supplementary Table S3.

\section{Affective Speech Recognition (ASR)}

Subjects were played a recording of four sentences of neutral content (eg 'The boy went to the store') with eight different emotional intonations (angry, disgusted, fearful, happy, lustful, neutral, sad, and surprised) (Hollander et al, 2007). Each emotional intonation was repeated six times for a total of 48 sentences with a fixed time interval for presentation and subject scoring. Subjects indicated the emotion by pointing to the corresponding word.

\section{Reading the Mind in the Eyes Test (RMET)}

Subjects were presented with 36 pictures of the eye region of different people, and instructed to choose which of four 
alternative labels best described what the person might be thinking or feeling (Baron-Cohen et al, 2001).

\section{Olfactory Identification}

The 'Sniffin' Sticks' smell identification test is a commercially available test of olfaction (Burghard Medical Technology, Wedel, Germany), which uses 12 pen-like devices, each containing a single odorant (Hummel et al, 2007). The devices were uncapped one at a time for $3 \mathrm{~s}$ and then placed $1-2 \mathrm{~cm}$ in front of the subject's nostrils. Subjects indicated the odorant among a field of four choices.

\section{Scripted Communication and Interaction Test}

The Scripted Communication and Interaction Test (SCIT) assessed change in communication domains in short-term treatment. The measure has two forms that are suitable for repeated administration with short intervals (in the order of weeks). A trained examiner conducts a structured 15-20-min conversational interaction using explicit and scripted prompts that focus on the following domains: social awareness and response to others; verbal and nonverbal responsiveness to the examiner; initiation of communication;

Table I Baseline Characteristics

\begin{tabular}{|c|c|}
\hline Characteristics & Mean (SD), $N=19$ \\
\hline Age, years & $23.4(5.1)$ \\
\hline Weight, kg & $79.5(19.6)$ \\
\hline $\mathrm{BMI}, \mathrm{kg} / \mathrm{m}^{2}$ & $25.7(5.5)$ \\
\hline Full scale IQ (Wechsler) ${ }^{\mathrm{a}}$ & $100(14.5)$ \\
\hline Verbal IQ & $98(17.4)$ \\
\hline Performance IQ & $10 \mid(\mid 1.1)$ \\
\hline ADOS total score & $12(4.8)$ \\
\hline Communication score & $3(1.7)$ \\
\hline Reciprocal social interaction score & $6(2.4)$ \\
\hline Communication+social interaction score & $10(3.7)$ \\
\hline Vineland II Adaptive Behavior score & $62(12.9)$ \\
\hline Adaptive behavior composite & $63(12.5)$ \\
\hline Communication & $65(21.8)$ \\
\hline Daily living skills & $66(9.6)$ \\
\hline Socialization & $65(13.0)$ \\
\hline ABC-full total score & $27(19.9)$ \\
\hline Irritability & $3(3.9)$ \\
\hline Lethargy/social withdrawal & $9(6.7)$ \\
\hline Stereotypic behavior & $3(2.6)$ \\
\hline Hyperactivity & $9(9.3)$ \\
\hline Inappropriate speech & $3(2.7)$ \\
\hline CGI-Severity & $4^{\mathrm{b}}(0.8)$ \\
\hline
\end{tabular}

Abbreviations: ABC, Aberrant Behavior Checklist; ADOS, Autism Diagnostic Observation Schedule; BMI, body mass index; IQ, intelligence quotient; SD, standard deviation; WASI, Wechsler Abbreviated Scale of Intelligence.

aFull-scale IQ was derived from subsets of WASI.

bModerately ill. conversational turn-taking; appropriateness during interaction; and emotional insight. At the end of the interview, the social and interactional skills are scored from 1 (behavior is absent, deficient, or odd) to 5 (typical for age) for each of the six domains; higher scores correspond to better social communication and interaction.

\section{Order of Assessments}

Owing to the number of measures, eye-tracking tasks and the ASR were conducted before and after drug administration (as a 2-h intravenous infusion), while the other assessments were performed after completion of the infusion (between 2 and $8 \mathrm{~h}$; CNS exposure was shown to remain constant up to $8 \mathrm{~h}$ (the last time-point assessed) post-infusion in the SAD study).

\section{Statistical Analysis}

Behavioral and questionnaire end points were analyzed using mixed model analysis of variance (ANOVA), with baseline measurement fitted in the model as a covariate if a pre-dose value was available. For each end point, the treatment difference (RG7713 - placebo) was estimated together with two-sided $90 \%$ confidence interval (CI). Effect sizes (ES; standardized mean differences) were calculated to allow comparison of effects across the different assessments and were considered as small (0.2), medium (0.5), or large $(0.8)$ (Cohen, 1988; Faraone, 2008).

In this crossover study, a sample size of approximately 20 subjects was considered sufficient to detect major tolerability and safety issues with RG7713 vs placebo. In addition, assuming a one-sided significance level of $5 \%$, a sample size of 18 would provide a power of more than $80 \%$ to detect an ES of 0.7 .

In line with the exploratory nature of this proof of mechanism study, no statistical control for type-I error from the multiple comparisons was pursued, and $p$-values should be interpreted with care, as descriptive weights of evidence rather than as confirmatory claims.

\section{RESULTS}

\section{Demographics}

Nineteen subjects with a diagnosis of autistic disorder (DSMIV-TR) were randomized between December 2011 and February 2013 to receive study drug and placebo in Sequence 1 (9 subjects) and Sequence 2 (10 subjects) (Supplementary Figure S1). All subjects completed the study. One subject was wrongly administered placebo during Period 2 after already receiving placebo during Period 1; this subject's data from Period 2 were excluded from the per-protocol analysis population. Another subject was wrongly administered study drug and placebo in reverse order; this subject's data were assigned to the treatment actually received and included in all analysis populations.

Subjects had a mean (SD) age of 23 (5.1) years, full scale IQ of 100 (14.5), VABS-II score of 62 (12.9), and CGI-Severity score of 4 (0.8), that is, were moderately ill. Further details of baseline characteristics are presented in Table 1. 


\section{Pharmacokinetics}

Plasma concentrations following the intravenous infusion of $20 \mathrm{mg}$ RG7713 over $2 \mathrm{~h}$ were comparable in adult male subjects with ASD (mean \pm SD concentration of $129 \pm 64.4 \mathrm{ng} / \mathrm{ml}$ at $2 \mathrm{~h}$ post-dose; and $45.6 \pm 12.9 \mathrm{ng} / \mathrm{ml}$ at $8 \mathrm{~h}$ post-dose) and healthy subjects in the SAD study $(130 \pm 16.8 \mathrm{ng} / \mathrm{ml}$ at $2 \mathrm{~h}$ post-dose and $46.9 \pm 8.44$ at $8 \mathrm{~h}$ post-dose; Supplementary Table S1).

\section{Efficacy}

Eye-tracking results showed that administration of RG7713 was associated with a large and statistically significant increase in biological motion orienting preference $(\mathrm{ES}=0.8$, $p=0.047$ ) and a non-significant improvement in the composite score $(\mathrm{ES}=0.2, p=0.29$ ) (Table 2). RG7713 was associated with a significantly reduced ability to detect lust in ASR $(E S=-0.8, p=0.03)$ and a non-significant reduction in the ability to detect fear $(\mathrm{ES}=-0.7, p=0.07)$. However, when all eight individual emotion subscales were combined into an overall ASR performance score, the change was nonsignificant ( $E S=-0.1, p=0.59$ ) (Table 2). Treatment with RG7713 was also associated with small, non-significant improvements in RMET performance, olfactory identification, and overall assessment of ASD with the CGI-I (RG7713: mean $\pm \mathrm{SD}=3 \pm 0.8$, minimally improved vs placebo: mean $\pm S D=4 \pm 0.5$, no change). A summary of results for all efficacy end points is provided in Table 2; results for other efficacy endpoints did not reach statistical significance.

\section{Safety}

Four AEs were reported in five subjects after treatment with RG7713, but not after placebo: dizziness $(n=2)$, disturbance in attention $(n=1)$, infusion site rash $(n=1)$, and anxiety $(n=1)$. All AEs reported (13 AEs in 10 subjects) were of mild or moderate severity, with the moderate AEs (2 AEs in 2 subjects) all reported during the placebo treatment period. Most AEs (10/13) resolved within 1 day of onset, and all resolved within 2 weeks. There were no serious AEs reported, no early terminations, and no clinically relevant changes in laboratory parameters, ECG, or vital signs were observed (Supplementary Table S4).

Post-dose anxiety levels measured by the STAI showed small improvements from baseline. However, no treatment differences were observed (RG7713 period pre-dose: mean $\pm \mathrm{SD}=31 \pm 7.8, \quad n=18 ; \quad \mathrm{RG7713}$ period post-dose: mean change from baseline $\pm \mathrm{SD}=-2 \pm 4.8$ points, $n=18$, $v s$ placebo period pre-dose: mean $\pm \mathrm{SD}=31 \pm 8.4, n=19$; placebo post-dose: mean change $\pm S D=-4 \pm 8.1$ points, $n=19)$.

\section{DISCUSSION}

We used exploratory biomarkers and behavioral assessments comprising eye-tracking paradigms, ASR, RMET, and olfactory identification along with the SCIT to investigate the effects of the novel, potent and highly selective V1a receptor antagonist, RG7713, on measures associated with communication and social cognition in high functioning adult subjects with ASD. The subjects in this study presented

diagnostic profiles, and impairments in RMET performance and olfaction identification similar to those of subjects with ASD reported in previous studies (Hus and Lord, 2014; Baron-Cohen et al, 2001; Suzuki et al, 2003; Bennetto et al, 2007; Galle et al, 2013; Ashwin et al, 2014; Wicker et al, 2016), but displayed higher ASR performance (57\% vs $\sim 41 \%$ ) than previously reported (Hollander et al, 2007). Although statistically significant effects were limited to increased orienting preference for biological motion in eye tracking and decreased ability to detect lust in ASR, this study provides preliminary evidence of a positive effect of RG7713 on social cognition. No safety signals associated with administration of RG7713 were identified.

The goal of this study was to gather evidence for V1a receptor antagonism as a potential treatment for deficits in social communication in ASD. Targeting complex behavioral domains such as social cognition and communication in ASD in a single-dose, proof-of-mechanism study is a challenging but necessary first step. Typical rating scales used in ASD target relatively heterogeneous groups of behaviors and were not originally developed to sensitively assess social communication or more narrow components of social responsiveness in response to a single dose of a drug, and were therefore considered unsuitable as primary pharmacodynamic variables in this study.

We expected that any therapeutic effects of RG7713 could be identified and quantified more accurately as effects on the component processes underlying social cognition and communication-analogous to results reported with singledose administration of oxytocin (Andari et al, 2010). Thus, we sought to identify effects of RG7713 with exploratory behavioral biomarkers and behavioral assessments relating to these component processes, combined with higher-order behavioral and clinical ratings. These biomarkers and assessments included eye-tracking paradigms, as a measure of attunement to and extraction of socially relevant information, and ASR and RMET, as measures of the ability to detect and process socially relevant information in human communication. We also explored effects on olfaction as a sensory modality assumed to play a role in social interaction, and a novel clinical assessment, the SCIT, to directly evaluate separate domains of social communication.

The observed effects of RG7713 treatment on eye tracking in this study were mostly subtle and their interpretation and extrapolation to behavioral outcomes is challenging. While the overall effects on eye tracking (composite score) suggested an improvement in abnormal eye gaze patterns, statistical significance was not reached. The largest signal $(E S=0.8)$ was seen in the biomotion paradigm in which subjects administered RG7713 oriented more often (orienting preference) to biological information. If replicated, this may suggest that RG7713 could have positive effects on a behaviorally 'primitive' attentional response to biologically relevant information (Klin et al, 2009; Simion et al, 2008). It is important to note that reduced attention to biological motion is one of the most highly replicated biomarker findings in ASD (Chita-Tegmark, 2016). Such reductions in social attention have been shown to be specific to ASD, as they are not seen in subjects with other developmental disabilities (Klin et al, 2009; Pierce et al, 2011). Indeed, this marker has been proposed by some as a possible screening test for ASD risk. It is theorized that reduced attention to 
Table 2 Efficacy Results

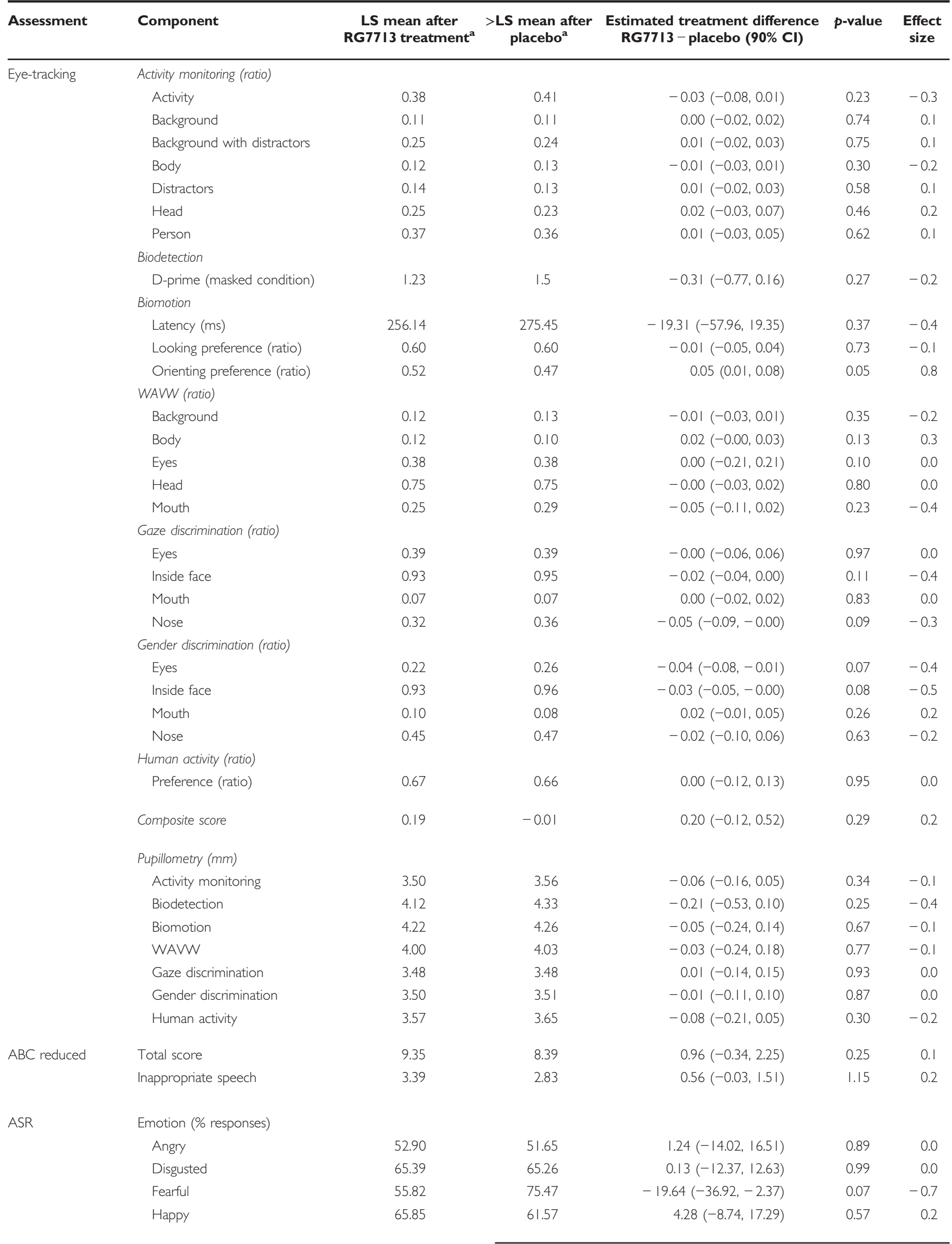


Table 2 Continued

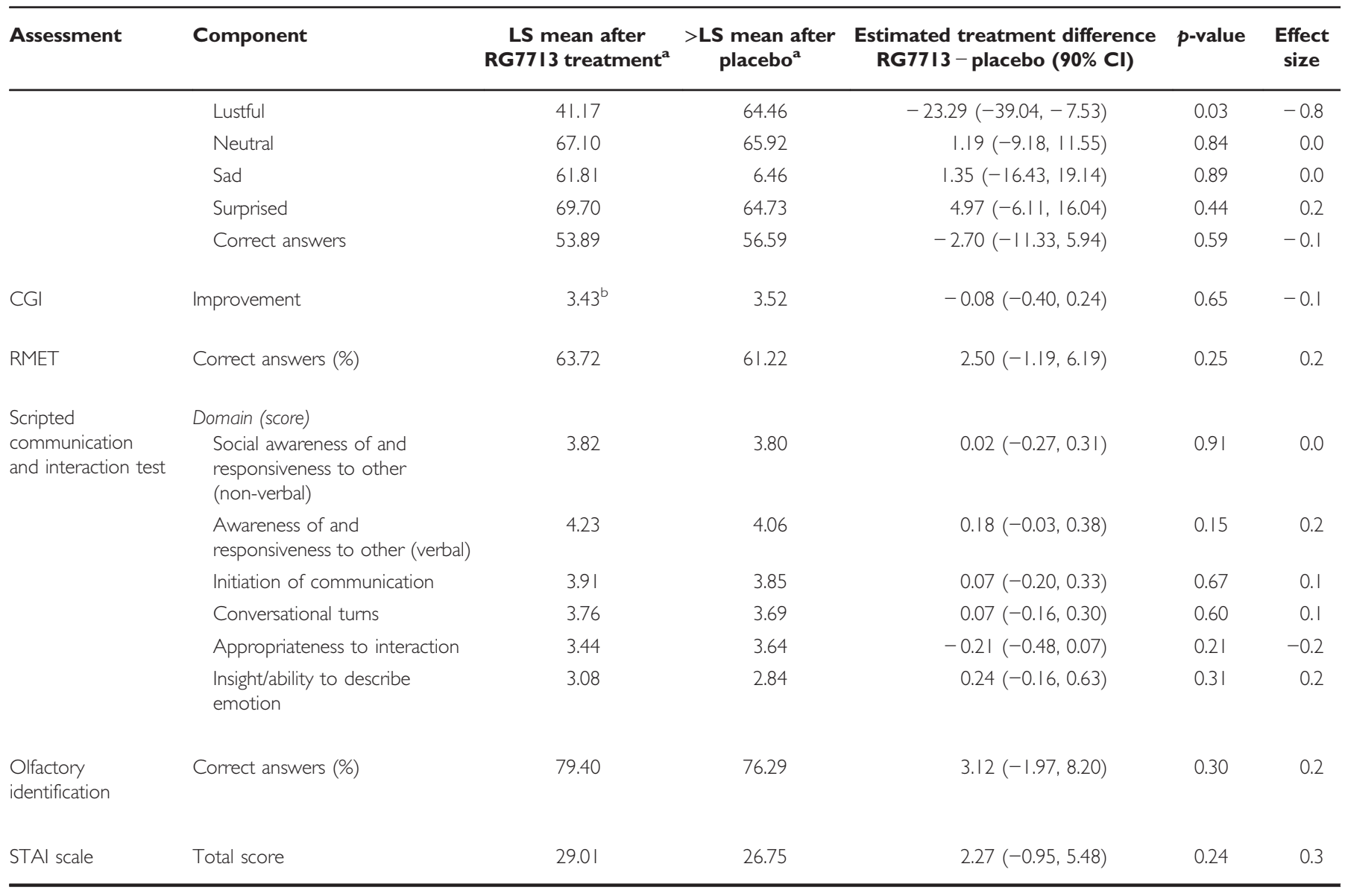

Abbreviations: ABC, Aberrant Behavior Checklist; ASR, affective speech recognition; CGl, Clinical Global Impressions scale; LS, least squared; Cl, confidence interval; ms, milliseconds; STAI, State-Trait Anxiety Inventory; RMET, reading the mind in the eyes test; WAVW, Who's Afraid of Virginia Woolf?

${ }^{a}$ Behavioral and questionnaire end points were analyzed using mixed model analysis of variance (ANOVA), with baseline measurement fitted in the model as a covariate if a pre-dose value was available.

$b_{3}=$ mildly ill; $4=$ moderately ill.

biological motion, especially prominent in testing paradigms of more complex and active social scenes, may create a cascading effect leading to impaired social responsiveness. Andari et al, (2010) evaluated eye tracking during face scanning in sex and gaze discrimination tasks in a comparable population of high functioning young adults with $\operatorname{ASD}(N=13$, mean age $=26$ years, age range $=17-39$ years); after treatment with a single dose of intranasal oxytocin, an improvement in visual scanning of faces and the eyes in gaze direction $(E S=0.6)$, and gender discrimination tasks $(E S=0.5)$ was observed. The effects of RG7713 on comparable measures in our study were weaker, and the biomotion paradigm on which the strongest effect was observed was not performed by Andari et al (2010). The different effects observed with oxytocin and RG7713 in the eye-tracking tasks could indicate that the vasopressin and oxytocin systems modulate different aspects of the process of acquisition of socially relevant information, rather than having simply antagonistic effects as is sometimes assumed.

There were small improvements with RG7713 in the identification of emotions in the RMET and identification of smells in the olfactory identification test; performance in both of these tests is impaired in patients with ASD (BaronCohen et al, 2001; Rozenkrantz et al, 2015). While the RMET and olfactory identification results observed in this study were not statistically significant, they may suggest improvements in social communication. Interestingly, we observed a slight worsening of the total performance in the ASR, largely driven by a decreased sensitivity to recognize fear and lust. Such an effect is consistent with a potential anxiolytic effect of a V1a receptor antagonist, bringing subjects' performance closer to levels observed in healthy volunteers (del Valle Rubido et al, 2016; F. Hoffmann-La Roche, 2014).

Results of studies in rodents and non-human primates indicate that increases in vasopressin receptor expression enhance social recognition (Liu et al, 2001), cooperative behavior, monogamous and con-specific bonding (Insel et al. 1994; Young et al, 1997) and social communication measured by flank marking and scent marking (Ferris et al, 1984, 1985; Albers et al, 1986; Irvin et al, 1990; Winslow and Insel, 1991; Hennessey et al, 1992), and that differences in neuroanatomical distribution of vasopressin V1a receptors can influence social organization and pair-bonding behavior (Young et al, 1999; Lim et al, 2004; Walum et al, 2008; 
Albers, 2012; Caldwell and Albers 2016). Thus, it could be argued that a vasopressin receptor agonist, but not antagonist, may be beneficial to treat ASD. However, vasopressin has also been shown to increase brain response to offensive aggression in rodents (Delville et al, 1996; Ferris et al, 1997; Bester-Meredith et al, 1999; Caldwell and Albers, 2004) and to increase maternal aggression (Winslow and Insel, 1991; Bosch, 2011; Bosch and Neumann, 2010). In humans, intranasal vasopressin administration was shown to increase threat perception in healthy volunteers (Thompson et al, 2004) to impair emotion recognition in males (Uzefovsky et al, 2012) and to modulate social behavior by increasing response to socially threatening stimuli, measured with BOLD magnetic resonance imaging during a face matching task (Zink et al, 2010). This may be the result of the effects of vasopressin on a neural network that combines the subgenual and supragenual cingulate, and amygdala through negative feedback loops (Zink et al, 2010). These findings along with the results of this study support the concept that vasopressin receptor antagonism could have a prosocial effect by ameliorating aggressive behavior and reducing threat perception, which would be beneficial for treatment of ASD.

We used a crossover design to control for inter-patient variability and improve the likelihood of identifying significant effects. Nevertheless, the study is limited by the small sample size, single drug dose, short timeframe for assessments (the assessment period was completed within $8 \mathrm{~h}$ of drug infusion) and potential selection bias (subjects were all high functioning adult males, with ABC-Irritability subscale scores $\leqslant 13$ ). In addition, a large number of statistical comparisons were performed without correction for multiplicity, which should be taken into account when interpreting the results.

Overall, the observed effects of RG7713 were quantitatively small, and the paucity of statistically significant results may suggest that the study was underpowered to detect them: the study was designed with a sample size sufficient to detect medium/large effect sizes, but most of the trends observed were of small/medium effects. Although the 8-h efficacy assessment window was supported by the drug exposure data (CSF concentrations remained at approximately the same level during that time, as measured in the SAD study), some effects may only emerge with longer continuous treatment, and may not be apparent yet, or only partially so, in a single-dose study. In addition, to simplify analyses, data were analyzed in aggregate, for example, a single total score was used for each eye-tracking outcome measure despite the presence of multiple assessments and embedded experimental conditions within tasks, potentially diminishing statistical power. Nonetheless, this study adds to the evidence that administration of single doses of potential therapeutic compounds for ASD can result in subtle but possibly meaningful changes in biomarkers and behavioral assessments of social cognition, and may constitute an informative approach to establish confidence in a therapeutic target early in clinical development.

In conclusion, we present initial evidence that treatment with the V1a receptor antagonist, RG7713, provides subtle improvements in social communication surrogates such as eye tracking, ASR, RMET, and olfaction identification, in adults with high-functioning ASD. These preliminary findings of the effects of a single $20 \mathrm{mg}$ dose and the use of these biomarkers support further clinical exploration of V1a receptor antagonism as a therapeutic approach to treat core symptoms in ASD.

\section{FUNDING AND DISCLOSURE}

Medical writing and submission support were provided by Charlie Hunt and Michael Maddalena of Watermeadow Medical, an Ashfield company, part of UDG Healthcare plc, funded by F. Hoffmann-La Roche. This study (ClinicalTrials. gov number: NCT01474278) was funded by F. Hoffmann-La Roche. The study drug, RG7713, is not commercially available and was provided by F. Hoffmann-La Roche. EH has acted as a consultant for Roche, and has received research grants from Roche, Curemark, Coronado Biosciences, Forest, Simons Foundation, Foundation for Prader Willi Research, Orphan Products Division of the Food and Drug Administration, and has intellectual property relating to oxytocin and autism. JTM has served as a consultant for Roche and Dart Neuroscience, has received research grants from Roche, and has received study drug from Shire and AstraZeneca. FS has received research funding from Roche and Janssen Pharmaceuticals. LSc has served as a consultant to Bracket, MedAdvante, Roche, Neuren and Coronado. DU, MVR, JN, LB, OK, LSq, CG, HK, and $\mathrm{PF}$ are full-time employees of F. Hoffmann-La Roche.

\section{ACKNOWLEDGMENTS}

EH, JTM, FS and LSc were trial investigators and contributed significantly to the conduct of the study and acquisition of clinical data. FS designed the eye-tracking experiments and analyzed the eye-tracking data, blinded to participant and experimental randomization information. LSq performed the statistical analyses. DU and LB designed the study, and together with MVR supervised its execution and analyses of the data. All authors were involved in review and interpretation of the data, preparing the first draft of the report, and providing further comments and revisions. All authors approved the final version of the report and take full responsibility for the content.

\section{REFERENCES}

Albers HE (2012). The regulation of social recognition, social communication and aggression: vasopressin in the social behavior neural network. Horm Behav 61: 283-292.

Albers HE, Pollock J, Simmons WH, Ferris CF (1986). A V1-like receptor mediates vasopressin-induced flank marking behavior in hamster hypothalamus. J Neurosci 6: 2085-2089.

Aman MG, Singh NN, Stewart AW, Field CJ (1985). The aberrant behavior checklist: a behavior rating scale for the assessment of treatment effects. Am J Ment Defic 89: 485-491.

American Psychiatric Association (2013). Diagnostic and Statistical Manual of Mental Disorders. 5th edn. (DSM-5). American Psychiatric Association: Arlington, VA.

Andari E, Duhamel JR, Zalla T, Herbrecht E, Leboyer M, Sirigu A (2010). Promoting social behavior with oxytocin in highfunctioning autism spectrum disorders. Proc Natl Acad Sci USA 107: 4389-4394. 
Ashwin C, Chapman E, Howells J, Rhydderch D, Walker I, Baron-Cohen S (2014). Enhanced olfactory sensitivity in autism spectrum conditions. Mol Autism 5: 53.

Baron-Cohen S, Leslie AM, Frith U (1985). Does the autistic child have a 'theory of mind'? Cognition 21: 37-46.

Baron-Cohen S, Wheelwright S, Hill J, Raste Y, Plumb I (2001). The 'Reading the Mind in the Eyes' test revised version: a study with normal adults, and adults with Asperger syndrome or high-functioning autism. J Child Psychol Psychiatry 42: 241-251.

Baxter AJ, Brugha TS, Erskine HE, Scheurer RW, Vos T, Scott JG (2015). The epidemiology and global burden of autism spectrum disorders. Psychol Med 45: 601-613.

Bennetto L, Kuschner ES, Hyman SL (2007). Olfaction and taste processing in autism. Biol Psychiatry 62: 1015-1021.

Bester-Meredith JK, Young LJ, Marler CA (1999). Species differences in paternal behavior and aggression in peromyscus and their associations with vasopressin immunoreactivity and receptors. Horm Behav 36: 25-38.

Bosch OJ (2011). Maternal nurturing is dependent on her innate anxiety: the behavioral roles of brain oxytocin and vasopressin. Horm Behav 59: 202-212.

Bosch OJ, Neumann ID (2010). Vasopressin released within the central amygdala promotes maternal aggression. Eur J Neurosci 31: 883-891.

Centers for Disease Control (2014). Prevalence of autism spectrum disorder among children aged 8 years-autism and developmental disabilities monitoring network, 11 sites, United States, 2010. MMWR Surveill Summ 63: 1-21.

Caldwell HK, Albers HE (2004). Effect of photoperiod on vasopressin-induced aggression in Syrian hamsters. Horm Behav 46: 444-449.

Caldwell HK, Albers HE (2016). Oxytocin, vasopressin, and the motivational forces that drive social behaviors. Curr Top Behav Neurosci 27: 51-103.

Chita-Tegmark M (2016). Social attention in ASD: a review and meta-analysis of eye-tracking studies. Res Dev Disabil 48: 79-93.

Cohen J (1988). Statistical Power Analysis for the Behavioral Sciences. Lawrence Erlbaum: Hillsdale, NJ.

del Valle Rubido M, Hollander E, McCracken J, Shic F, Scahill L, Noeldeke J et al (2016). A multi-center, non-drug study to explore the relationship between exploratory biomarkers and functional dimensions in individuals with autistic disorder or Asperger's syndrome Submitted for publication.

Delville Y, Mansour KM, Ferris CF (1996). Testosterone facilitates aggression by modulating vasopressin receptors in the hypothalamus. Physiol Behav 60: 25-29.

F. Hoffmann-La Roche (2014). Data on file, BP28421.

F. Hoffmann-La Roche (2015). Data on file, BP22583.

Faraone SV (2008). Interpreting estimates of treatment effects: implications for managed care. Pharm Ther 33: 700-711.

Ferris CF, Albers HE, Wesolowski SM, Goldman BD, Luman SE (1984). Vasopressin injected into the hypothalamus triggers a stereotypic behavior in golden hamsters. Science 224: 521-523.

Ferris CF, Pollock J, Albers HE, Leeman SE (1985). Inhibition of flank-marking behavior in golden hamsters by microinjection of a vasopressin antagonist into the hypothalamus. Neurosci Lett 55: 239-243.

Ferris CF, Melloni RH Jr, Koppel G, Perry KW, Fuller RW, Delville $Y$ (1997). Vasopressin/serotonin interactions in the anterior hypothalamus control aggressive behavior in golden hamsters. $J$ Neurosci 17: 4331-4340.

Frith C, Frith U (2005). Theory of mind. Curr Biol 15: R644-R645.

Galle SA, Courchesne V, Mottron L, Frasnelli J (2013). Olfaction in the autism spectrum. Perception 42: 341-355.

Guy W (1976). Clinical Global Impression Scale. The ECDEU Assessment Manual for Psychopharmacology-Revised Volume DHEW Publ No ADM 76 338: 218-222.
Grimwood S, Hartig PR (2009). Target site occupancy: emerging generalizations from clinical and preclinical studies. Pharm Ther 122: 281-301.

Hennessey AC, Whitman DC, Albers HE (1992). Microinjection of arginine-vasopressin into the periaqueductal gray stimulates flank marking in Syrian hamsters (Mesocricetus auratus). Brain Res 569: $136-140$.

Hollander E, Bartz J, Chaplin W, Phillips A, Sumner J, Soorya L et al (2007). Oxytocin increases retention of social cognition in autism. Biol Psychiatry 61: 498-503.

Hummel T, Kobal G, Gudziol H, Mackay-Sim A (2007). Normative data for the 'Sniffin'Sticks' including tests of odor identification, odor discrimination, and olfactory thresholds: an upgrade based on a group of more than 3,000 subjects. Eur Arch Oto-RhinoLaryngol 264: 237-243.

Hus V, Lord C (2014). The Autism Diagnostic Observation Schedule, Module 4: revised algorithm and standardized severity scores. J Autism Dev Disord 44: 1996-2012.

Insel TR, Wang ZX, Ferris CF (1994). Patterns of brain vasopressin receptor distribution associated with social organization in microtine rodents. J Neurosci 14: 5381-5392.

International Conference on Harmonisation (1996). ICH Harmonised Tripartite Guideline: Guideline for Good Clinical Practice E6 (R1) Step 4. 10-Jun-1996.

Irvin RW, Szot P, Dorsa DM, Potegal M, Ferris CF (1990). Vasopressin in the septal area of the golden hamster controls scent marking and grooming. Physiol Behav 48: 693-699.

Ji NY, Findling RL (2015). An update on pharmacotherapy for autism spectrum disorder in children and adolescents. Curr Opin Psychiatry 28: 91-101.

Kaiser MD, Delmolino L, Tanaka JW, Shiffrar M (2010). Comparison of visual sensitivity to human and object motion in autism spectrum disorder. Autism Res 3: 191-195.

Kim SJ, Young LJ, Gonen D, Veenstra-VanderWeele J, Courchesne R, Courchesne $\mathrm{E}$ et al (2002). Transmission disequilibrium testing of arginine vasopressin receptor 1A (AVPR1A) polymorphisms in autism. Mol Psychiatry 7: 503-507.

Klin A, Jones W, Schultz R, Volkmar F, Cohen D (2002). Visual fixation patterns during viewing of naturalistic social situations as predictors of social competence in individuals with autism. Arch Gen Psychiatry 59: 809-816.

Klin A, Lin DJ, Gorrindo P, Ramsay G, Jones W (2009). Two-yearolds with autism orient to non-social contingencies rather than biological motion. Nature 459: 257-261.

Lim MM, Hammock EA, Young LJ (2004). The role of vasopressin in the genetic and neural regulation of monogamy. J Neuroendocrinol 16: 325-332.

Liu Y, Curtis JT, Wang ZX (2001). Vasopressin in the lateral septum regulates pair bond formation in male prairie voles (Microtus ochrogaster). Behav Neurosci 115: 910-919.

Lord C, Rutter M, Goode S, Heemsbergen J, Jordan H, Mawhood L et al (1989). Autism diagnostic observation schedule: a standardized observation of communicative and social behavior. J Autism Dev Disord 19: 185-212.

Meyer-Lindenberg A, Kolachana B, Gold B, Olsh A, Nicodemus KK, Mattay V et al (2009). Genetic variants in AVPR1A linked to autism predict amygdala activation and personality traits in healthy humans. Mol Psychiatry 14: 968-975.

Pierce K, Conant D, Hazin R, Stoner R, Desmond J (2011). Preference for geometric patterns early in life as a risk factor for autism. Arch Gen Psychiatry 68: 101-109.

Posner K, Brown GK, Stanley B, Brent DA, Yershova KV, Oquendo MA et al (2011). The Columbia-Suicide Severity Rating Scale: initial validity and internal consistency findings from three multisite studies with adolescents and adults. Am J Psychiatry 168: 1266-1277.

Ratni H, Rogers-Evans M, Bissantz C, Grundschober C, Moreau JL, Schuler F et al (2015). Discovery of highly selective brain- 
penetrant vasopressin 1a antagonists for the potential treatment of autism via a chemogenomic and scaffold hopping approach. J Med Chem 58: 2275-2289.

Rozenkrantz L, Zachor D, Heller I, Plotkin A, Weissbrod A, Snitz K et al (2015). A mechanistic link between olfaction and autism spectrum disorder. Curr Biol 25: 1904-1910.

Shic F, Bradshaw J, Klin A, Scassellati B, Chawarska K (2011). Limited activity monitoring in toddlers with autism spectrum disorder. Brain Res 1380: 246-254.

Simion F, Regolin L, Bulf H (2008). A predisposition for biological motion in the newborn baby. Proc Natl Acad Sci USA 105: 809-813.

Sparrow SS, Cicchetti DV, Balla DA (2005). Vineland Adaptive Behavior Scales-Manual. NCS Pearson: Minneapolis, MN.

Spielberger CD, Gorsuch RL (1983). State-Trait Anxiety Inventory for Adults: Manual and Sample: Manual, Instrument and Scoring Guide. Consulting Psychologists Press: Palo Alto, CA.

Suzuki Y, Critchley HD, Rowe A, Howlin P, Murphy DG (2003). Impaired olfactory identification in Asperger's syndrome. J Neuropsychiatry Clin Neurosci 15: 105-107.

Thompson R, Gupta S, Miller K, Mills S, Orr S (2004). The effects of vasopressin on human facial responses related to social communication. Psychoneuroendocrinol 29: 35-48.

Uzefovsky F, Shalev I, Israel S, Knafo A, Ebstein RP (2012). Vasopressin selectively impairs emotion recognition in men. Psychoneuroendocrinol 37: 576-580.

Walum H, Westberg L, Henningsson S, Neiderhiser JM, Reiss D, Igl W et al (2008). Genetic variation in the vasopressin receptor la gene (AVPR1A) associates with pair-bonding behavior in humans. Proc Natl Acad Sci USA 105: 14153-14156.

Wassink TH, Piven J, Vieland VJ, Pietila J, Goedken RJ, Folstein SE et al (2004). Examination of AVPR1a as an autism susceptibility gene. Mol Psychiatry 9: 968-972.

Wechsler D (1999). Wechsler Abbreviated Scale of Intelligence. Psychological Corporation: San Antonio, TX.

Wicker B, Monfardini E, Royet JP (2016). Olfactory processing in adults with autism spectrum disorders. Mol Autism 7: 4.

Winslow J, Insel TR (1991). Vasopressin modulates male squirrel monkeys' behavior during social separation. Eur J Pharmacol 200: 95-101.
World Medical Association (2008). Declaration of Helsinki. Ethical principles for medical research involving human subjects Last amended by the 59th WMA AssemblyOctober 2008.

Yang SY, Cho SC, Yoo HJ, Cho IH, Park M, Yoe J et al (2010). Family-based association study of microsatellites in the 5 flanking region of AVPR1A with autism spectrum disorder in the Korean population. Psychiatry Res 178: 199-201.

Yirmiya N, Rosenberg C, Levi S, Salomon S, Shulman C, Nemanov L et al (2006). Association between the arginine vasopressin 1a receptor (AVPR1a) gene and autism in a familybased study: mediation by socialization skills. Mol Psychiatry 11: 488-494.

Young LJ, Nilsen R, Waymire KG, MacGregor GR, Insel TR (1999). Increased affiliative response to vasopressin in mice expressing the V1a receptor from a monogamous vole. Nature 400: $766-768$

Young LJ, Winslow JT, Nilsen R, Insel TR (1997). Species differences in V1a receptor gene expression in monogamous and nonmonogamous voles: behavioral consequences. Behav Neurosci 111: 599-605.

Zink CF, Stein JL, Kempf L, Hakimi S, Meyer-Lindenberg A (2010). Vasopressin modulates medial prefrontal cortex-amygdala circuitry during emotion processing in humans. J Neurosci 30: 7017-7022.

This work is licensed under a Creative Commons Attribution-NonCommercial-NoDerivs $\quad 4.0$ International License. The images or other third party material in this article are included in the article's Creative Commons license, unless indicated otherwise in the credit line; if the material is not included under the Creative Commons license, users will need to obtain permission from the license holder to reproduce the material. To view a copy of this license, visit http://creativecommons.org/licenses/by-nc-nd/4.0/

(C) The Author(s) 2017

Supplementary Information accompanies the paper on the Neuropsychopharmacology website (http://www.nature.com/npp) 